\title{
Bioconvection Flow of MHD Viscous Nanofluid in the Presence of Chemical Reaction and Activation Energy
}

\author{
Muhammad Imran Asjad (D), ${ }^{1}$ Muhammad Zahid, ${ }^{1}$ Fahd Jarad (D), ${ }^{2,3,4}$ \\ and Abdullah M. Alsharif ${ }^{5}$ \\ ${ }^{1}$ Department of Mathematics, University of Management and Technology, Lahore 54770, Pakistan \\ ${ }^{2}$ Department of Mathematics, Cankaya University, Etimesgut, Ankara, Turkey \\ ${ }^{3}$ Department of Mathematics, King Abdul Aziz University, Jeddah, Saudi Arabia \\ ${ }^{4}$ Department of Medical Research, China Medical University Hospital, China Medical University, Taichung, Taiwan \\ ${ }^{5}$ Department of Mathematics and Statistics, College of Science, Taif University, Taif 21944, Saudi Arabia \\ Correspondence should be addressed to Fahd Jarad; fahd@cankaya.edu.tr
}

Received 14 October 2021; Revised 22 November 2021; Accepted 31 December 2021; Published 7 February 2022

Academic Editor: Muhammad Irfan

Copyright (c) 2022 Muhammad Imran Asjad et al. This is an open access article distributed under the Creative Commons Attribution License, which permits unrestricted use, distribution, and reproduction in any medium, provided the original work is properly cited.

\begin{abstract}
Enhancement of heat transfer due to stretching sheets can be appropriately controlled by the movement of the nanofluids. The concentration and settling of the nanoparticles in the viscous MHD fluid and bioconvection are addressed. In this scenario, the fluid flow occurring in the presence of a normal and uniform magnetic field, thermal radiation, and chemical reaction is taken into account. For the two-dimensional flow with heat and mass transfer, five dependent variables and three independent variables constitute the system of partial differential equations. For this purpose, similarity functions are involved to convert these equations to corresponding ODEs. Then, the Runge-Kutta method with shooting technique is used to evaluate the required findings with the utilization of MATLAB script. The fluid velocity becomes slow against the strength of the magnetic parameter. The temperature rises with the parameter of Brownian motion and thermophoresis. The bioconvection Lewis number diminishes the velocity field. Compared with the existing literature, the results show satisfactory congruences.
\end{abstract}

\section{Introduction}

The convoluted and quick process in massive machinery and little gadgets has produced a significant problem of thermal imbalance. Varied extraneous techniques like fins and fans are used; however, their utility is restricted because of their giant size. In 1995, the scientist Choi and Eastman [1] introduced that the nanosized particles mixed in the fluid called nanofluid have more capacity of heat transfer as compared with fluid without nanosized particles. Das et al. [2] explained the recent and future applications of fluid involving nanosized particles. Khan et al. [3] using the shooting method analyzed flow features of Williamson nanofluid influenced by variable viscosity depending on temperature and Lorentz force past an inclined nonlinear extending surface. Koo and Kleinstreuer [4] described influences of convection, conduction, viscous dissipation, and thermal transportation on nanofluid flow in a microchannel. Sui et al. [5] introduced the CattaneoeChristov model with double diffusion to analyze the influence of slip velocity, Brownian motion, and variable viscosity on the transportation of an upper convected Maxwell nanofluid through stretching sheet. Imran et al. [6] determined an unsteady stream of Maxwell fluid through an accelerated exponentially vertical surface with influences of radiation, Newtonian heating, MHD, and slip condition taken into account. Khan et al. [7] investigated the flow of micropolar base nanofluid through stretching sheet with thermal radiation and magnetic dipole. Sheikholeslami and Rokni [8] scrutinized magnetic field impacts on the thermal transport rate in a nanofluid. Seyyedi et al. [9] analyzed the entropy generation for $\mathrm{Cu}$-water nanofluid having a semi-annulus porous wavy cavity in the presence of a magnetic field. Molana et al. [10] discussed the characteristics of heat transfer and natural 
convection of nanofluid past a porous cavity with a constant inclined magnetic field. Dogonchi et al. [11] explained the characteristics of natural convection and magnetic field on nanofluid flow through porous medium with effects of Hartmann number, Rayleigh number, and Darcy number taken into account. Shaw et al. [12] scrutinized the impact of nonlinear thermal and entropy generation on Casson nanofluid flow with rotating disk and also described the brain function. Chamkha et al. [13] explained MHD nanofluid flow through cavity using the control-volumebased finite element method with effects of natural convection, thermal radiation, and shape factor of nanoparticles taken into account. Dogonchi et al. [14] numerically introduced the importance of the Cattaneo-Christov theory of heat conduction through triangular semicircular heater with viscosity dependent on the magnetic field. Seyyedi et al. [15] described the entropy generation and natural convection heat transfer of $\mathrm{Cu}$-water nanoliquid through the hexagonal cavity. Sadeghi et al. [16] analyzed the thermal behavior of magnetic buoyancy-driven flow in ferrofluid-filled wavy enclosure furnished with two circular cylinders.

Stretching sheets because of their wide applications like plastic film, metal drawing and spinning, glass fiber, paper processing, and heat moving have become an important topic in the past decades. Recently, some researchers investigated the magnetohydrodynamic flow with the different effects such as viscous dissipation and chemical reaction using stretching sheet (see Ismail et al. [17], Rajput et al. [18], Swain et al. [19], Reddy et al. [20], Jat and Chand [21], Sajid and Hayat [22], Ishak [23], Abd El-Aziz [24], Makinde [25], and Goud et al. [26]).

Bioconvection described the phenomena in which living microorganisms denser than water swim upward in suspensions. These microorganisms pile up in the layer of the upper surface and lower surface becomes less dense. The microorganisims fall down due to unstability of density distribution. Bioconvection has applications in biological systems and biotechnology such as purifying cultures, enzyme biosensors, and separating dead and living cells [27]. Raees et al. [28] examined the unsteady stream of bioconvection mixed nanofluid having gyrostatic motile microorganisms through a horizontal channel. Siddiqa et al. [29] numerically studied the bioconvection flow of nanofluid having mass and thermal transportation along with gyrotactic microorganisms through a curved vertical cone.
Abbasi et al. [30] introduced the bioconvection stream of viscoelastic nanofluid because of gyrotactic microorganisms past a rotating extending disk having zero mass flux and convective boundary condition and also described the relatable parameters influences on velocity, temperature, local density, Sherwood number, and Nusselt number in detail. Chu et al. [31] analyzed the stream of bioconvection MHD fluid through extending sheet with the significance of motile microorganisms, activation energy, thermophoresis diffusion, Brownian motion, and chemical reaction taken into account. Henda et al. [32] examined the magnetized bioconvection flow of fluid past an extending cylinder with thermal radiation, activation energy, and heat source. Khan et al. [33] scrutinized bioconvection stream of viscous nanofluid through (cone, wedge, and plate) multiple geometries with effects of heat flux, cross-diffusion, and Cattaneo-Christov.

Inspired by the above literature survey, our interest pertains to extending the results of Goud et al. [26] to investigate a more general problem, including bioconvection of nanofluid transportation with the effects of chemical reaction and radiation to avoid probable settling of nanoentities. The connotation of such meaningful attributes can be a useful extension, and the results can be utilized for desired effective thermal transportation in the heat exchanger of various technological processes.

\section{Problem Formulation}

Here, we considered steady incompressible magnetohydrodynamic nanofluid flow through exponentially stretching sheet along the $x$-axis and $y$-axis taken to be normal with velocity $\widetilde{U}_{w}=a_{0} e^{x / l}$ as shown in Figure 1 . A magnetic field is applied to the flow region and acts in the $y$-direction. A mild diffusion of microorganisms and nanoparticles is set in the fluid. Thermal radiation is considered, and bioconvection takes place because of microorganisms' movement. The fluid velocity for two-dimensional flow is $\widetilde{u}, \widetilde{v}$.

Under the above conditions, the governing equations are as follows $[20,26]$. Continuity equation is as follows:

$$
\widetilde{u}_{x}+\widetilde{v}_{y}=0
$$

momentum equation is as follows:

$$
\widetilde{u} \widetilde{u}_{x}+\widetilde{v} \widetilde{u}_{y}=v \widetilde{u}_{y y}-\frac{\sigma}{\rho}\left(B_{0}^{2} \widetilde{u}\right)+\frac{1}{\rho}\left[g \beta \rho\left(1-\widetilde{C}_{\infty}\right)\left(\widetilde{T}-\widetilde{T}_{\infty}\right)-g\left(\rho_{p}-\rho_{f}\right)\left(\widetilde{C}-\widetilde{C}_{\infty}\right)-g \gamma\left(\rho_{m}-\rho_{f}\right)\left(\widetilde{N}-\widetilde{N}_{\infty}\right)\right]
$$

energy equation is as follows: 


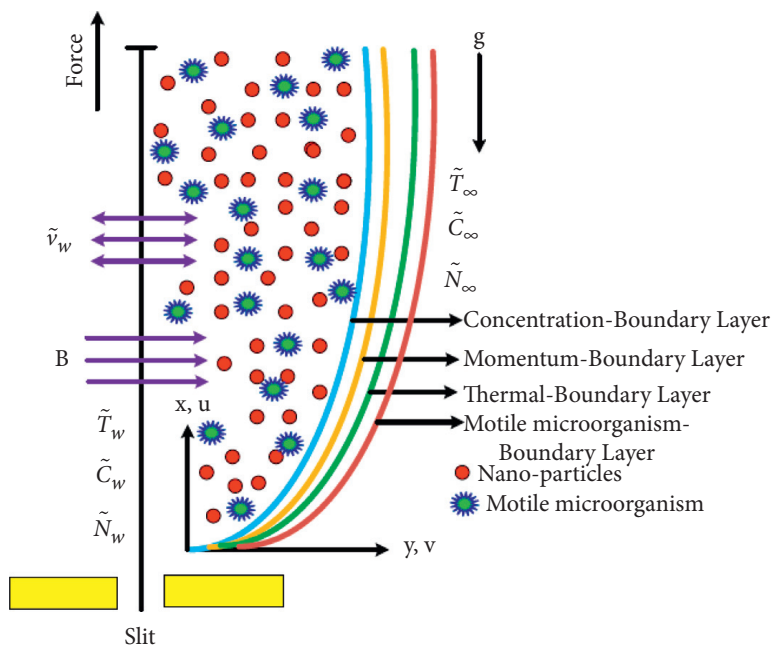

Figure 1: Geometry of the problem.

$\widetilde{u} \widetilde{T}_{x}+\widetilde{v} \widetilde{T}_{y}=\alpha \widetilde{T}_{y y}-\frac{1}{\rho C_{p}} \frac{\partial q_{r}}{\partial y}+\tau\left(D_{B} \widetilde{T}_{y} \widetilde{C}_{y}+\frac{D_{T}}{\widetilde{T}_{\infty}}\left(\widetilde{T}_{y}\right)^{2}\right)$

concentration equation is as follows:

$$
\widetilde{u} \widetilde{C}_{x}+\widetilde{v} \widetilde{C}_{y}=D \widetilde{C}_{y y}-K_{r}\left(\widetilde{C}-\widetilde{C}_{\infty}\right)-K_{r}^{2}\left(\widetilde{C}-\widetilde{C}_{\infty}\right)\left(\frac{\widetilde{T}}{\widetilde{T}_{\infty}}\right)^{n} \exp \left(\frac{-E a}{k \widetilde{T}}\right)+\frac{D_{T}}{\widetilde{T}_{\infty}} \widetilde{T}_{y y}
$$

bioconvection equation is as follows:

with constraints

$$
\widetilde{u} \tilde{N}_{x}+\widetilde{v} \tilde{N}_{y}+d W_{c} \frac{\partial}{\partial y}\left(\frac{\widetilde{N}}{\Delta \widetilde{C}} \widetilde{C}_{y}\right)=\widetilde{N}_{y y} D_{n}
$$

$$
\begin{aligned}
& \tilde{u}=\widetilde{U}_{w}, \widetilde{v}=0, \widetilde{T}=\widetilde{T}_{w}, \widetilde{C}=\widetilde{C}_{w}, \tilde{N}=\widetilde{N}_{w} \text { at } y \longrightarrow 0 \\
& \widetilde{u} \longrightarrow 0, \widetilde{T} \longrightarrow \widetilde{T}_{\infty}, \widetilde{C} \longrightarrow \widetilde{C}_{\infty}, \tilde{N} \longrightarrow \widetilde{N}_{\infty} \text { as } y \longrightarrow \infty
\end{aligned}
$$

Now, introducing

$$
\widetilde{U}_{w}=a_{0} e^{x / l}, \widetilde{T}_{w}=\widetilde{T}_{\infty}+\widetilde{T}_{0} e^{x / 2 l}, \widetilde{C}_{w}=\widetilde{C}_{\infty}+\widetilde{C}_{0} e^{x / 2 l}, \widetilde{N}_{w}=\widetilde{N}_{\infty}+\widetilde{N}_{0} e^{x / 2 l}
$$

under the Rosseland approximation $q_{r}$ [26], equation (3) can be written as 


$$
\widetilde{u} \widetilde{T}_{x}+\widetilde{v} \widetilde{T}_{y}=\widetilde{T}_{y y}\left(\alpha+\frac{16 \sigma^{*} T_{\infty}^{3}}{\rho C_{p} 3 k_{1}}\right)+\tau\left(D_{B} \widetilde{T}_{y} \widetilde{C}_{y}+\frac{D_{T}}{\widetilde{T}_{\infty}}\left(\widetilde{T}_{y}\right)^{2}\right)
$$

Introducing similarity transformation,

$$
\begin{aligned}
& \eta=y \sqrt{\frac{a_{0}}{2 \nu \ell}} e^{x / 2 l}, \widetilde{u}=a_{0} e^{x / l} f^{\prime}(\eta), \widetilde{v}=-\sqrt{\frac{a_{0} \nu}{2 \ell}} e^{x / 2 l}\left[f(\eta)+\eta f^{\prime}(\eta)\right], \\
& \psi=\sqrt{2 \nu L a_{0}} f(\eta) e^{x / 2 l}, \widetilde{T}=\widetilde{T}_{\infty}+\widetilde{T}_{0} e^{x / 2 l}, \widetilde{C}=\widetilde{C}_{\infty}+\widetilde{C}_{0} e^{x / 2 l}, \widetilde{N}=\widetilde{N}_{\infty}+\widetilde{N}_{0} e^{x / 2 l}
\end{aligned}
$$

In view of the above appropriate relations, equation (1) is satisfied identically and equations (2)-(5), respectively, become

$$
\begin{aligned}
& f^{\prime \prime \prime}-M f^{\prime}-2 f^{\prime 2}+f f^{\prime \prime}+\lambda(\theta-N r \phi-R b \chi)=0, \\
& \left(1+\frac{4}{3} K\right) \theta^{\prime \prime}+\operatorname{Pr} f \theta^{\prime}-\operatorname{Pr} \theta f^{\prime}+\theta^{\prime}\left(N b \phi^{\prime}+N t \theta^{\prime}\right)=0, \\
& \phi^{\prime \prime}+\left[f \phi^{\prime}-\operatorname{Cr} \phi-\phi f^{\prime}-\sigma_{m} \phi(1+\delta \theta)^{n} \exp \left(\frac{-E}{1+\delta \theta}\right)\right] S c+\frac{N t}{N b} \theta^{\prime \prime}=0, \\
& \chi^{\prime \prime}(\xi)+\operatorname{Lb} \operatorname{Pr} f(\xi) \chi^{\prime}(\xi)-\operatorname{LbPr} f^{\prime}(\xi) \chi(\xi)-\operatorname{Pe}\left(\sigma_{1} \phi^{\prime \prime}(\xi)+\chi(\xi) \phi^{\prime \prime}(\xi)+\chi^{\prime}(\xi) \phi^{\prime}(\xi)\right)=0,
\end{aligned}
$$

and the constraints reduce to

$$
\begin{gathered}
f^{\prime}(0)=1, f(0)=0, \phi(0)=1, \theta(0)=1, \chi(0)=1 \text {, at } \eta=0, \\
f^{\prime}(\infty) \longrightarrow 0, \phi(\infty) \longrightarrow 0, \theta(\infty) \longrightarrow 0, \chi(\infty) \longrightarrow 0 \text {. as } \eta \longrightarrow \infty .
\end{gathered}
$$

The associated parameters are

$$
\begin{aligned}
M & =\frac{2 \sigma B_{0}^{2} \ell}{\rho \widetilde{U}_{w}}, \\
\operatorname{Pr} & =\frac{\nu}{\alpha}, \\
\lambda & =\frac{\left(1-C_{\infty}\right) \beta g\left(T_{w}-T_{\infty}\right) 2 \ell}{\widetilde{U}_{w}^{2}}, \\
N r & =\frac{\left(\rho_{p}-\rho_{f}\right)\left(C_{w}-C_{\infty}\right)}{\beta\left(1-C_{\infty}\right) \rho\left(T_{w}-T_{\infty}\right)}, \\
R b & =\frac{\left(\rho_{m}-\rho_{f}\right) \gamma\left(N_{w}-N_{\infty}\right)}{\rho\left(1-C_{\infty}\right) \beta\left(T_{w}-T_{\infty}\right)},
\end{aligned}
$$

$$
\begin{aligned}
N t & =\frac{\tau D_{T}\left(T_{w}-T_{\infty}\right)}{\nu T_{\infty}}, \\
N b & =\frac{\tau D_{B}\left(C_{w}-C_{\infty}\right)}{\nu}, \\
\sigma_{m} & =\frac{2 K_{r}^{2} \ell}{\widetilde{U}_{w}}, \\
\delta & =\frac{\left(T_{w}-T_{\infty}\right)}{T_{\infty}}, \\
K & =\frac{4 \sigma^{*} T_{\infty}^{3}}{k^{*} K}, \\
\sigma_{1} & =\frac{N_{\infty}}{N_{w}-N_{\infty}},
\end{aligned}
$$


Table 1: Comparison of $\theta^{\prime}(0)$ with changed values of $K, M$, and Pr.

\begin{tabular}{ccccccc}
\hline$K$ & $M$ & Pr & Ishak [23] & Goud et al. [26] & Bidin and Nazar [34] & Our results \\
\hline & & 1.0 & 0.9548 & 0.954784 & 0.9547 & 1.4714 \\
0.9548106 \\
0.0 & 0 & 2.0 & 1.4715 & 1.471462 & 1.8691 & 1.4714540 \\
& & 3.0 & 1.8691 & 1.869073 & 2.8690688 \\
& & 5.0 & 2.5001 & 2.500111 & 3.6603693 \\
1.0 & 10.0 & 3.6604 & 3.660346 & 0.8615086 \\
& 1.0 & 1.0 & 0.8611 & 0.861097 & 0.5313112 \\
\end{tabular}

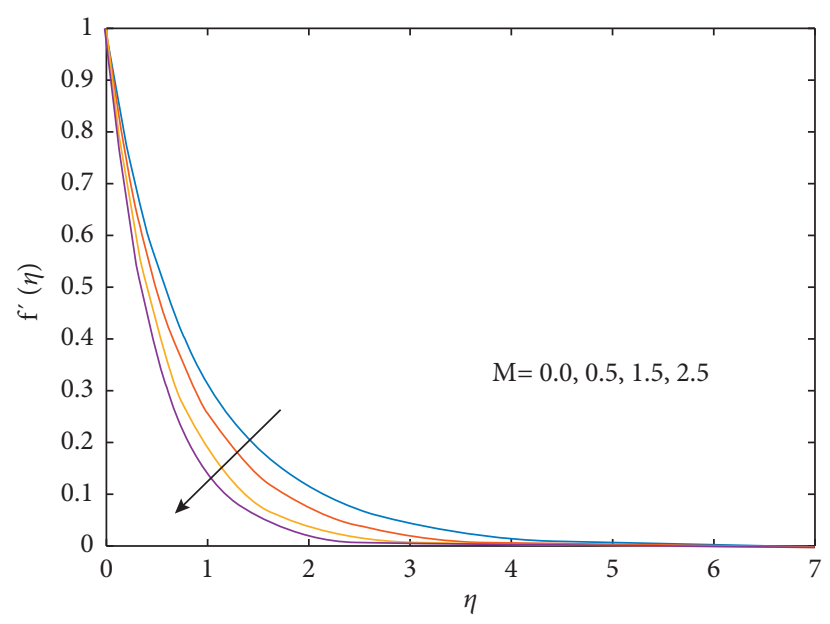

Figure 2: Influences of $M$ on $f^{\prime}$.

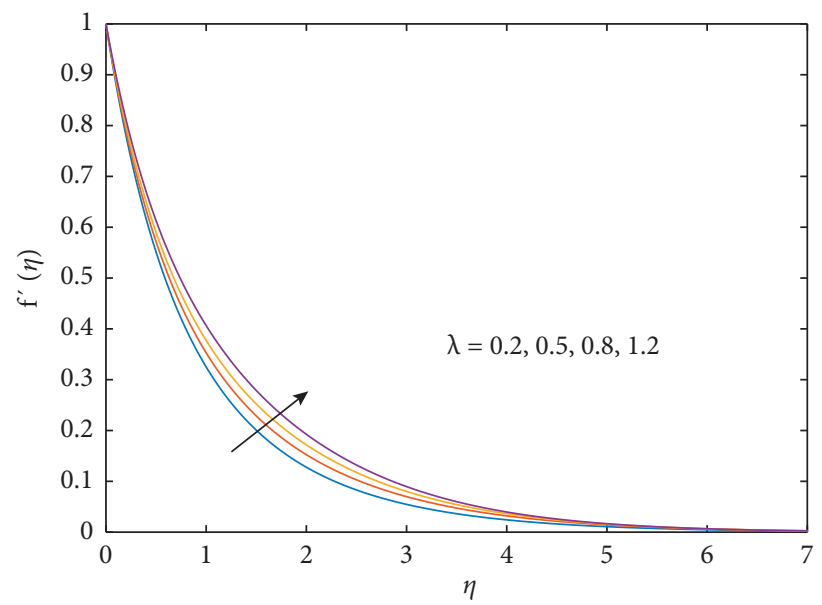

Figure 3: Influences of $\lambda$ on $f^{\prime}$.

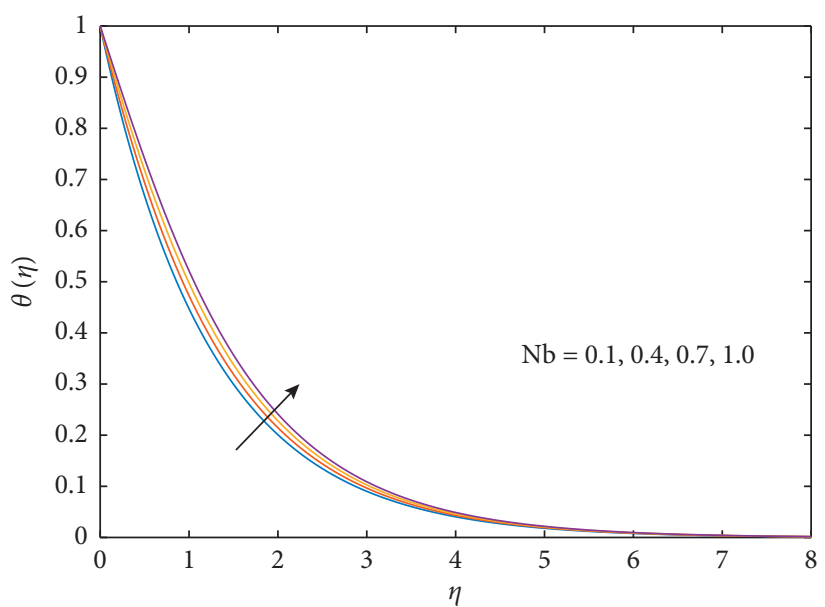

Figure 4: Influences of $\mathrm{Nb}$ on $\theta$. 


$$
\begin{gathered}
E=\frac{E a}{k T_{\infty}}, \\
S c=\frac{\nu}{D^{\prime}}, \\
L b=\frac{\alpha}{D_{n}}, \\
P e=\frac{d W_{c}}{D_{n}}, \\
C r=\frac{2 \ell K_{r}}{\widetilde{U}_{w}},
\end{gathered}
$$

where $M$ is the magnetic field parameter, Pr is the Prandtl number, $\lambda$ is the mixed convection parameter, $\mathrm{Nr}$ is the buoyancy ratio number, $\mathrm{Rb}$ is the bioconvection Rayleigh number, $\mathrm{Nt}$ is the thermopherosis diffusion factor, $\mathrm{Nb}$ is the Brownian factor, $\sigma_{m}$ denotes the dimensionless reaction rate, $\delta$ is used as the temperature distinction parameter, $K$ is the radiation parameter, $\sigma_{1}$ is the bioconvective difference parameter, $E$ means the nondimensional energy activation, Sc is the Schmidt number, $\mathrm{Lb}$ is the bioconvection Lewis number, $\mathrm{Pe}$ is the peclet number, and $\mathrm{Cr}$ is the chemical reaction parameter.

The wall shear stress, thermal flux, and mass flux, respectively, are given as

$$
\tau_{w}-\mu\left(\frac{\partial \widetilde{u}}{\partial y}\right)_{y=0}=0, q_{w}+k\left(\frac{\partial \widetilde{T}}{\partial y}\right)_{y=0}=0, j_{w}+D\left(\frac{\partial \widetilde{c}}{\partial y}\right)_{y=0}=0 .
$$

$C_{f}$ (skin friction), $N u_{x}$ (Nusselt number), and $S h_{x}$ (sherwood number) in dimensionless form are

$C_{f}=\frac{f^{\prime \prime}(0)}{\sqrt{2 \mathrm{Re}_{x}}}, N u_{x}=-\left(\sqrt{\mathrm{Re}_{x}}\right) \theta^{\prime}(0), \quad S h_{x} \operatorname{Re}^{-1 / 2}=-\phi^{\prime}(0)$.

\section{Results and Discussion}

Physical meanings of the final nondimensional formulation of time-independent MHD flow of nanofluid due to stretch of an exponential sheet in the presence of chemical reaction along the boundary constraints are solved numerically. Table 1 contains results for $-\theta^{\prime}(0)$ (Nusselt number). Comparison of the results indicates acceptable agreement to validate this numeric procedure. In Figure 2, the velocity of the flow seems to be reduced significantly when magnetic parameter $M(0.0 \leq M \leq 2.5)$ is increased because high values of magnetic field parameter improve the contradictory force known as Lorentz force. The improvement of

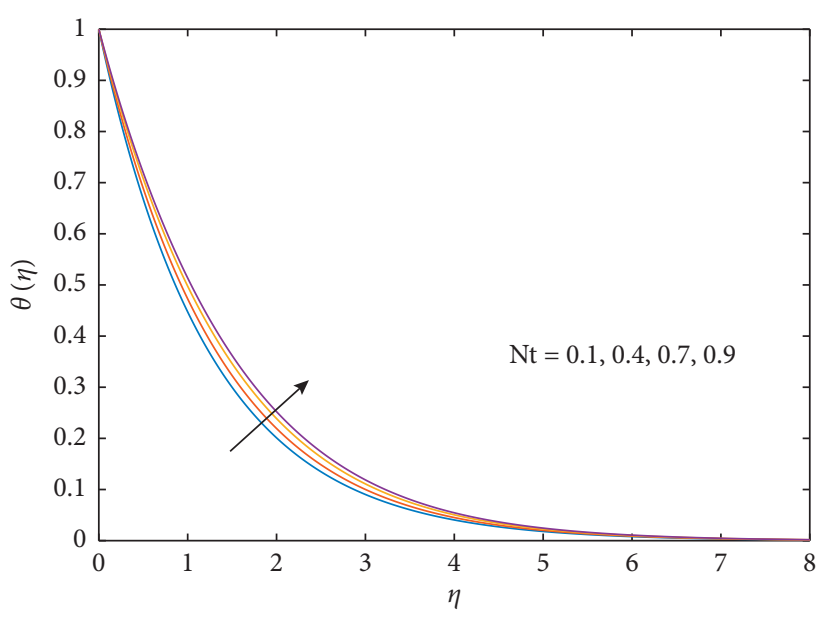

Figure 5: Influence of $\mathrm{Nt}$ on $\phi$.

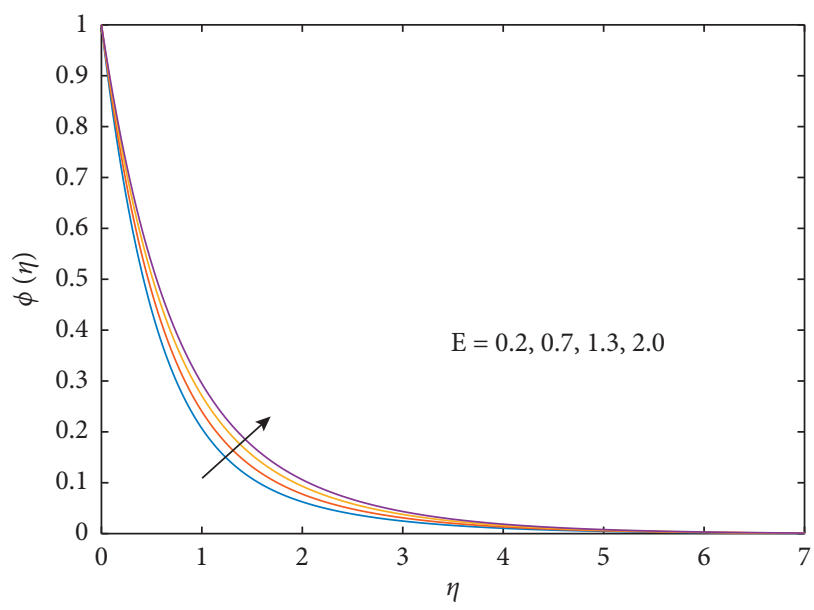

Figure 6: Influences of $E$ on $\phi$.

mixed convection parameter $\lambda$ causes to boost the flow velocity $f^{\prime}(\eta)$ as shown in Figure 3 . From Figures 4 and 5, significant rising behavior of $\theta(\eta)$ is noticed with an enhanced value of Brownian motion parameter $\mathrm{Nb}$ and thermophoresis parameter Nt. The fast random motion of nanoparticles characterized by larger $\mathrm{Nb}$ is responsible for enhanced heat transfer to raise $\theta(\eta)$. Similarly, the higher $\mathrm{Nt}$ means a greater thermophoretic effect which moves the nanoparticles hotter regime to the colder one and increases the thermal distribution. The similarly larger value of $E$ provides strength to $\phi(\eta)$ as depicted in Figure 6. Figure 7 displays the decrement in $\phi(\eta)$ due to the larger value of chemical reaction, and the chemical reaction becomes faster to recede nanoparticles concentration. The bioconvection Rayleigh $\mathrm{Rb}$ and parameter are responsible for given direct increment to $\chi(\eta)$ as demonstrated in Figure 8. 


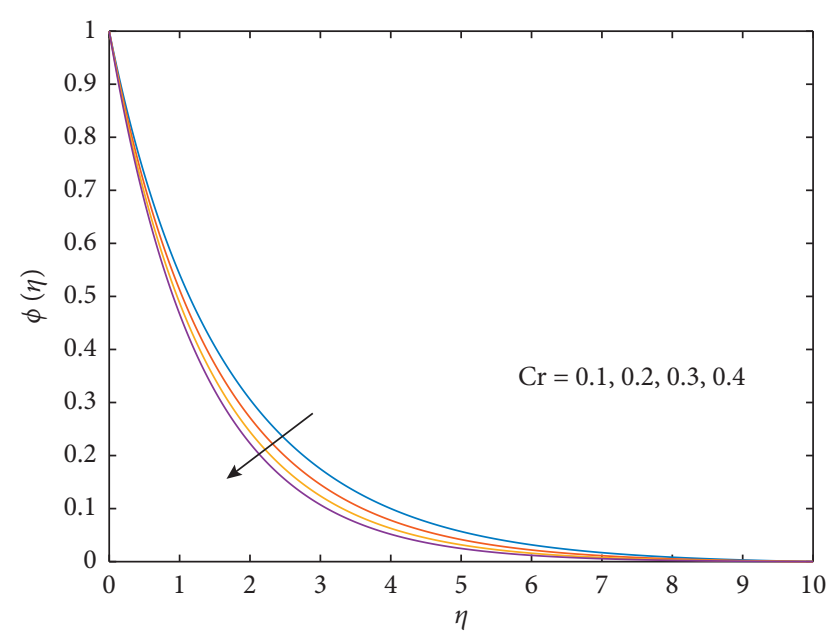

Figure 7: Influences of $\mathrm{Cr}$ on $\phi$.

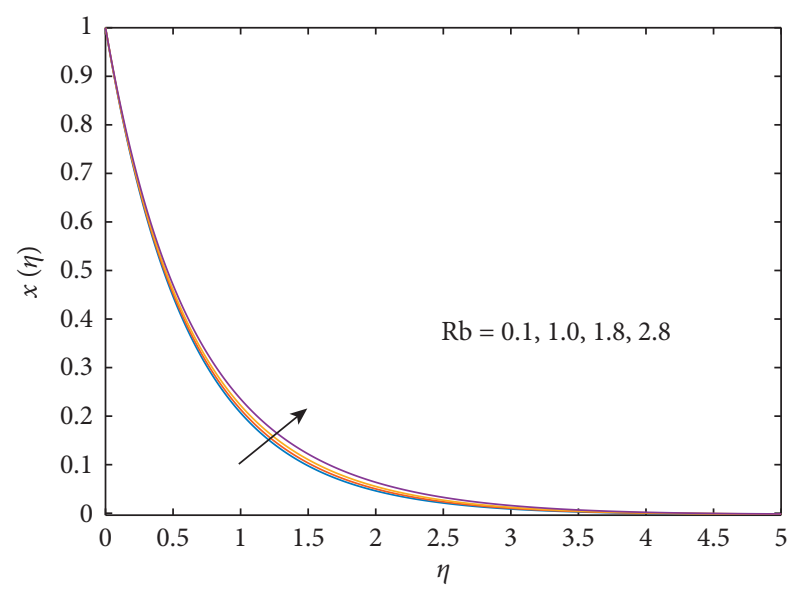

Figure 8: Influences of $\mathrm{Rb}$ on $\chi$.

\section{Conclusions}

Theoretical and numeric analysis for magnetohydrodynamic of nanofluid owing to sudden stretched in an exponential sheet has been made in this communication. Effects of the emerging parameters are enumerated on the physical field, namely, velocity, temperature, and microorganisms distribution. Significant outcomes are summarized as follows:

(i) The velocity reduces with $M$ and boosts with $\lambda$

(ii) The conclusion of nanoparticles characterized by parameters $\mathrm{Nb}$ and $\mathrm{Nt}$ shows an increment in the temperature profile

(iii) Concentration recurses with $\mathrm{Cr}$ and is enhanced with $E$

(iv) Bioconvection parameter is increased with $\mathrm{Rb}$

\section{Nomenclature}

$\begin{array}{ll}B_{0}: & \text { Coefficient of magnetic field } \\ C: & \text { Concentration } \\ T: & \text { Temperature }\end{array}$

$N$ : Concentration of microorganisms

$N t: \quad$ Thermopherosis parameter

$(x, y)$ : Cartesian coordinates

$C r$ : Chemical reaction parameter

$(u, v)$ : Velocity components along $(x, y)$-axes

$\tau: \quad$ Heat capacity ratio

$\xi: \quad$ Similarity variable

$D_{T}$ : Thermophoretic diffusion coefficient

$\phi$ : Dimensionless concentration

$q_{r}: \quad$ Radiative heat flux

$\rho: \quad$ Density

$K_{r}^{2}$ : Chemical reaction rate constant

$\mu$ : Dynamic viscosity of the fluid

$K_{r}: \quad$ Rate of chemical reaction

$\sigma: \quad$ Electrical conductivity

$D_{B}$ : Brownian diffusivity

$\psi: \quad$ Stream function

K: $\quad$ Radiation parameter

$\delta: \quad$ Temperature distinction parameter

Sc: $\quad$ Schmidt number

$\lambda: \quad$ Mixed convection parameter

$U_{w}$ : $\quad$ Stretching velocity

v: $\quad$ Kinematic viscosity

Pr: Prandtl number

$\theta$ : Dimensionless temperature

Pe: Peclet number

$\chi$ : Dimensionless microorganism factor

$M: \quad$ Magnetic parameter

$\rho_{f}: \quad$ Density of nanofluid

$N r$ : Buoyancy ratio number

$\rho_{m}$ : Density of microorganisms particle

$R b$ : Bioconvection Rayleigh number

$\rho_{p}: \quad$ Density of nanoparticles

$N b$ : Brownian motion parameter

$\kappa: \quad$ Thermal conductivity

$n$ : $\quad$ Fitted rate constant parameter

$\alpha$ : Thermal diffusivity

g: $\quad$ Gravity

$\beta$ : Volumetric coefficient of thermal expansion

$E$ : Nondimensional activation energy

$\gamma: \quad$ Average volume of microorganism

$L b$ : Bioconvection Lewis number

$S h_{x}$ : Local Sherwood Number

$W_{c}$ : Maximum cell swimming speed

$\sigma_{m}$ : Dimensionless reaction rate

$D_{n}$ : Microorganisms diffusion coefficient

$\sigma_{1}$ : Bioconvection diffrrence parameter

$\sigma^{*}$ : Stefan Boltzman constant

$N u_{x}$ : Local Nusselt number

$C_{f}$ : Local skin friction number

$R e_{x}$ : Local Reynolds number.

\section{Data Availability}

The data used to support this study are included within this article. 


\section{Conflicts of Interest}

The authors declare that they have no known personal relationships or conflicts of interest that could have appeared to the work reported in this work.

\section{Authors' Contributions}

All authors contributed equally and significantly in writing this paper. All authors read and approved the manuscript.

\section{Acknowledgments}

This research was supported by Taif University Researchers Supporting Project Number TURSP-2020/96, Taif University, Taif, Saudi Arabia.

\section{References}

[1] S. U. Choi and J. A. Eastman, "Enhancing thermal conductivity of fluids with nanoparticles," Tech. Rep. 96:001707, Argonne National Laboratory, Argonne, IL, USA, 1995.

[2] S. K. Das, S. U. Choi, W. Yu, and T. Pradeep, Nanofluids: Science and Technology, John Wiley \& Sons, New York, NY, USA, 2007.

[3] M. Khan, M. Y. Malik, T. Salahuddin, and A. Hussian, "Heat and mass transfer of williamson nanofluid flow yield by an inclined lorentz force over a nonlinear stretching sheet," Results in Physics, vol. 8, pp. 862-868, 2018.

[4] J. Koo and C. Kleinstreuer, "Laminar nanofluid flow in microheat-sinks," International Journal of Heat and Mass Transfer, vol. 48, no. 13, pp. 2652-2661, 2005.

[5] J. Sui, L. Zheng, and X. Zhang, "Boundary layer heat and mass transfer with Cattaneo-Christov double-diffusion in upperconvected Maxwell nanofluid past a stretching sheet with slip velocity," International Journal of Thermal Sciences, vol. 104, pp. 461-468, 2016.

[6] M. A. Imran, M. B. Riaz, N. A. Shah, and A. A. Zafar, "Boundary layer flow of mhd generalized Maxwell fluid over an exponentially accelerated infinite vertical surface with slip and Newtonian heating at the boundary," Results in Physics, vol. 8, pp. 1061-1067, 2018.

[7] S. A. Khan, B. Ali, C. Eze et al., "Magnetic dipole and thermal radiation impacts on stagnation point flow of micropolar based nanofluids over a vertically stretching sheet: finite element approach," Processes, vol. 9, no. 7, p. 1089, 2021.

[8] M. Sheikholeslami and H. B. Rokni, "Simulation of nanofluid heat transfer in presence of magnetic field: a review," International Journal of Heat and Mass Transfer, vol. 115, pp. 1203-1233, 2017.

[9] S. M. Seyyedi, A. Dogonchi, R. Nuraei, D. Ganji, and M. Hashemi-Tilehnoee, "Numerical analysis of entropy generation of a nanofluid in a semi-annulus porous enclosure with different nanoparticle shapes in the presence of a magnetic field," The European Physical Journal Plus, vol. 134, no. 6, pp. 1-20, 2019.

[10] M. Molana, A. S. Dogonchi, T. Armaghani, A. J. Chamkha, D. D. Ganji, and I. Tlili, "Investigation of hydrothermal behavior of fe3o4-h2o nanofluid natural convection in a novel shape of porous cavity subjected to magnetic field dependent (mfd) viscosity," Journal of Energy Storage, vol. 30, p. 101395 , 2020.
[11] A. S. Dogonchi, S. M. Seyyedi, M. Hashemi-Tilehnoee, A. J. Chamkha, and D. D. Ganji, "Investigation of natural convection of magnetic nanofluid in an enclosure with a porous medium considering brownian motion," Case Studies in Thermal Engineering, vol. 14, p. 100502, 2019.

[12] S. Shaw, A. S. Dogonchi, M. K. Nayak, and O. D. Makinde, "Impact of entropy generation and nonlinear thermal radiation on Darcy-forchheimer flow of MnFe2O4-casson/water nanofluid due to a rotating disk: application to brain dynamics," Arabian Journal for Science and Engineering, vol. 45, no. 7, pp. 5471-5490, 2020.

[13] A. Chamkha, A. S. Dogonchi, and D. D. Ganji, "Magnetohydrodynamic nanofluid natural convection in a cavity under thermal radiation and shape factor of nanoparticles impacts: a numerical study using cvfem," Applied Sciences, vol. 8, no. 12, p. 2396, 2018.

[14] A. S. Dogonchi, M. Waqas, M. M. Gulzar, M. HashemiTilehnoee, S. M. Seyyedi, and D. D. Ganji, "Simulation of $\mathrm{Fe} 3 \mathrm{O} 4-\mathrm{H} 2 \mathrm{O}$ nanoliquid in a triangular enclosure subjected to Cattaneo-Christov theory of heat conduction," International Journal of Numerical Methods for Heat and Fluid Flow, vol. 29, no. 11, pp. 4430-4444, 2019.

[15] S. M. Seyyedi, A. S. Dogonchi, M. Hashemi-Tilehnoee, D. D. Ganji, and A. J. Chamkha, "Second law analysis of magneto-natural convection in a nanofluid filled wavy-hexagonal porous enclosure," International Journal of Numerical Methods for Heat and Fluid Flow, vol. 30, no. 11, pp. 48114836, 2020.

[16] M. S. Sadeghi, T. Tayebi, A. S. Dogonchi, M. K. Nayak, and M. Waqas, "Analysis of thermal behavior of magnetic buoyancy-driven flow in ferrofluid-filled wavy enclosure furnished with two circular cylinders," International Communications in Heat and Mass Transfer, vol. 120, p. 104951, 2021.

[17] N. S. A. Ismail, A. S. Abd Aziz, M. R. Ilias, and S. K. Soid, "Mhd boundary layer flow in double stratification medium," Journal of Physics: Conference Series IOP Publishing, vol. 1770, no. 1, p. 12045, 2021.

[18] G. R. Rajput, B. P. Jadhav, and S. N. Salunkhe, "Magnetohydrodynamics boundary layer flow and heat transfer in porous medium past an exponentially stretching sheet under the influence of radiation," Heat Transfer, vol. 49, no. 5, pp. 2906-2920, 2020.

[19] B. K. Swain, B. C. Parida, S. Kar, and N. Senapati, "Viscous dissipation and joule heating effect on mhd flow and heat transfer past a stretching sheet embedded in a porous medium," Heliyon, vol. 6, no. 10, p. e05338, 2020.

[20] N. N. Reddy, V. S. Rao, and B. R. Reddy, "Chemical reaction impact on mhd natural convection flow through porous medium past an exponentially stretching sheet in presence of heat source/sink and viscous dissipation," Case Studies in Thermal Engineering, vol. 25, p. 100879, 2021.

[21] R. N. Jat and G. Chand, "Mhd flow and heat transfer over an exponentially stretching sheet with viscous dissipation and radiation effects," Applied Mathematical Sciences, vol. 7, no. 4, pp. 167-180, 2013.

[22] M. Sajid and T. Hayat, "Influence of thermal radiation on the boundary layer flow due to an exponentially stretching sheet," International Communications in Heat and Mass Transfer, vol. 35, no. 3, pp. 347-356, 2008.

[23] A. Ishak, "Mhd boundary layer flow due to an exponentially stretching sheet with radiation effect," Sains Malaysiana, vol. 40, no. 4, pp. 391-395, 2011. 
[24] M. Abd El-Aziz, "Viscous dissipation effect on mixed convection flow of a micropolar fluid over an exponentially stretching sheet," Canadian Journal of Physics, vol. 87, no. 4, pp. 359-368, 2009.

[25] O. D. Makinde, "Free convection flow with thermal radiation and mass transfer past a moving vertical porous plate," International Communications in Heat and Mass Transfer, vol. 32, no. 10, pp. 1411-1419, 2005.

[26] B. Shankar Goud, P. Srilatha, P. Bindu, and Y. Hari Krishna, "Radiation effect on mhd boundary layer flow due to an exponentially stretching sheet," Advances in Mathematics: Scientific Journal, vol. 9, no. 12, pp. 10755-10761, 2020.

[27] A. M. Spormann, "Unusual swimming behavior of a magnetotactic bacterium," FEMS Microbiology Letters, vol. 45, no. 1, pp. 37-45, 1987.

[28] A. Raees, H. Xu, and S.-J. Liao, "Unsteady mixed nanobioconvection flow in a horizontal channel with its upper plate expanding or contracting," International Journal of Heat and Mass Transfer, vol. 86, pp. 174-182, 2015.

[29] S. Siddiqa, N. Gul-e-Hina, N. Begum, S. Saleem, and M. A. Reddy Gorla, "Numerical solutions of nanofluid bioconvection due to gyrotactic microorganisms along a vertical wavy cone," International Journal of Heat and Mass Transfer, vol. 101, pp. 608-613, 2016.

[30] A. Abbasi, F. Mabood, W. Farooq, and M. Batool, "Bioconvective flow of viscoelastic nanofluid over a convective rotating stretching disk," International Communications in Heat and Mass Transfer, vol. 119, p. 104921, 2020.

[31] Y.-M. Chu, M. I. Khan, N. B. Khan et al., "Significance of activation energy, bio-convection and magnetohydrodynamic in flow of third grade fluid (non-Newtonian) towards stretched surface: a buongiorno model analysis," International Communications in Heat and Mass Transfer, vol. 118, p. 104893, 2020.

[32] M. Ben Henda, H. Waqas, M. Hussain et al., "Applications of activation energy along with thermal and exponential spacebased heat source in bioconvection assessment of magnetized third grade nanofluid over stretched cylinder/sheet," Case Studies in Thermal Engineering, vol. 26, p. 101043, 2021.

[33] S. A. Khan, H. Waqas, S. M. R. S. Naqvi, M. Alghamdi, and Q. Al-Mdallal, "Cattaneo-christov double diffusions theories with bio-convection in nanofluid flow to enhance the efficiency of nanoparticles diffusion," Case Studies in Thermal Engineering, vol. 26, p. 101017, 2021.

[34] B. Bidin and R. Nazar, "Numerical solution of the boundary layer flow over an exponentially stretching sheet with thermal radiation," European Journal of Scientific Research, vol. 33, no. 4, pp. 710-717, 2009. 